\title{
Potent inhibitory effect of Foeniculum vulgare Miller extract on osteoclast differentiation and ovariectomy-induced bone loss
}

\author{
TAE-HO KIM ${ }^{1,2}$, HYUN-JU KIM ${ }^{1}$, SANG-HAN LEE ${ }^{4,5}$ and SHIN-YOON KIM ${ }^{1,3}$ \\ ${ }^{1}$ Skeletal Diseases Genome Research Center, Kyungpook National University Hospital, Daegu 700-412; \\ Departments of ${ }^{2}$ Medicine and ${ }^{3}$ Orthopedic Surgery, Kyungpook National University School of Medicine, \\ Daegu 700-422; ${ }^{4}$ Department of Food Science and Biotechnology, and ${ }^{5}$ Food and Bio-Industry \\ Research Institute, Kyungpook National University, Daegu 702-701, Republic of Korea
}

Received December 5, 2011; Accepted January 23, 2012

DOI: $10.3892 / \mathrm{ijmm} .2012 .950$

\begin{abstract}
Inhibition of osteoclast differentiation and bone resorption is considered an effective therapeutic approach to the treatment of postmenopausal bone loss. To find natural compounds that may inhibit osteoclastogenesis, we screened herbal extracts on bone marrow cultures. In this study, we found that an aqueous extract of Foeniculum vulgare Miller seed (FvMs) at low concentration, which has traditionally been used as a treatment for a variety of ailments, inhibits the osteoclast differentiation and bone resorptive activity of mature osteoclasts. We further investigated the effects of FvMs on ovariectomy (OVX)-induced bone loss using microcomputed tomography, biomechanical tests and serum marker assays for bone remodeling. Oral administration of FvMs $(30 \mathrm{mg}$ or $100 \mathrm{mg} / \mathrm{kg} /$ day) for 6 weeks had an intermediary effect on the prevention of femoral bone mineral density (BMD), bone mineral content (BMC), and other parameters compared to OVX controls. In addition, FvMs slightly decreased bone turnover markers that were accelerated by OVX. The boneprotective effects of FvMs may be due to suppression of an OVX-induced increase in bone turnover. Collectively, our findings indicate that FvMs have potential in preventing bone loss in postmenopausal osteoporosis by reducing both osteoclast differentiation and function.
\end{abstract}

Correspondence to: Dr Shin-Yoon Kim, Skeletal Diseases Genome Research Center, Kyungpook National University Hospital, Daegu 700-412, Republic of Korea

E-mail: syukim@knu.ac.kr

Professor Sang-Han Lee, Department of Food Science and Biotechnology, and Food and Bio-Industry Research Institute, Kyungpook National University, Daegu 702-701, Republic of Korea E-mail: sang@knu.ac.kr

Key words: Foeniculum vulgare, osteoclast, ovariectomy, bone loss resorption, bone

\section{Introduction}

Osteoporosis is a systemic bone disease characterized by a reduction in bone mass, disruption in bone microarchitecture, and a consequent increase in bone fragility. Adult bones are continuously remodeled through the formation of new bone tissue by osteoblasts and the resorption of old bone tissue by osteoclasts (1). In general, an imbalance in bone remodeling caused by increased bone resorption over bone formation leads to most adult skeletal diseases, including osteoporosis and rheumatoid arthritis (2). Therefore, enhanced activation of osteoclasts is the target for therapeutic intervention of pathological bone loss.

Current drugs for bone health include bisphosphonates, calcitonin, and estrogen, which prevent osteoclastic bone resorption, resulting in the maintenance of bone mass and a reduction in the number of fractures $(3,4)$; however, each of these drugs is associated with some adverse effects, such as hypercalcemia, increased risk of breast and endometrial cancer, and gastrointestinal intolerance for bisphosphonate $(5,6)$. Hence, it is necessary to develop naturally occurring compounds with fewer side effects that can substitute for or eliminate the drugs that are currently used.

Traditional medicines derived from plants have been used to prevent and treat osteoporosis in many countries, since they have fewer adverse reactions and are more suitable for long-term use than chemically synthesized medicines. To find natural compounds that may inhibit osteoclastogenesis, we screened herbal extracts and compounds on a bone marrow culture system. During our screening we observed that an aqueous extract of Foeniculum vulgare Miller (Fennel) seed (FvMs) has strong inhibitory effects on osteoclast differentiation and bone resorption in vitro. Fennel has shown numerous effects in vivo and in vitro, including anti-inflammatory (7) and antioxidant activity $(8,9)$; however, its beneficial effects on bone metabolism have not previously been evaluated. The present study was conducted to investigate the effect of Fennel extracts on osteoclast differentiation and function in vitro and possible beneficial effects on bone loss in an ovariectomized mouse model. 


\section{Materials and methods}

Preparation of an aqueous extract of Foeniculum vulgare Miller seed (FvMs). Seeds of Foeniculum vulgare Miller, harvested at a farm in Young Cheon, between October and November 2008, were identified by a senior staff member of the University. The sample was pulverized, and $\sim 200 \mathrm{~g}$ of powder was extracted with $1,000 \mathrm{ml}$ distilled water by an extractor (DWP-3800T; Separable Glass Pot, DaeWoong Co., Seoul, Korea) for $6 \mathrm{~h}$ at $60^{\circ} \mathrm{C}$. The extracts were filtrated, lyophilized, and then dissolved in appropriate buffers and adjusted to a concentration of $100 \mathrm{mg} / \mathrm{ml}$ for various in vitro and in vivo assays. The voucher specimen of the plant has been deposited in the Laboratory of Enzyme Biotechnology, Kyungpook National University, Daegu, Korea.

Osteoclast differentiation assay. To generate mouse-bonemarrow-derived osteoclasts, monocytes were isolated from the bone marrow of the tibia and femurs of 6-8 week-old ICR mice as previously described (10). Non-adherent bone marrow cells were seeded in 96 -well plates $\left(3 \times 10^{4}\right.$ cells/well) and cultured with $10 \mathrm{ng} / \mathrm{ml} \mathrm{M}-\mathrm{CSF}$ for 3 days. At this stage, the cells were considered bone-marrow-derived macrophages (BMMs). For osteoclastogenesis, BMMs were cultured with RANKL (20 ng/ml) and M-CSF (10 ng/ml) for 4-5 days in the presence or absence of sample. The tartrate-resistant acid phosphatase (TRAP) staining and its activity assay are described in our previous papers $(10,11)$. Cell viability was determined by the CellTiter $96^{\circledR}$ AQueous One Solution Cell Proliferation Assay kit (Promega, Madison, WI) following the manufacturer's instructions.

Reverse transcriptase-polymerase chain reaction analysis. Total RNA was prepared using TRI reagent according to the manufacturer's instructions. Total RNA $(2 \mu \mathrm{g})$ from each sample was reverse-transcribed with a SuperScript II Reverse Transcriptase (Invitrogen, Carlsbad, CA), and cDNA was amplified by PCR. The primers and PCR conditions for TRAP, Cathepsin K, MMP-9, GAPDH (12), c-Src (13), NFATc1, and DC-STAMP (14) have all been previously published. The PCR products were electrophoresed on a $1 \%$ agarose gel and the image was captured with an ImageQuant LAS 4000 documentation system (GE Healthcare, Piscataway, NJ).

Resorption pit formation. For the resorption pit assays, multinucleated osteoclasts were induced. Mouse BMM cells $\left(3 \times 10^{4}\right.$ cells/well) were seeded on bone slices (IDS Ltd., Boldon, UK) and treated with RANKL $(20 \mathrm{ng} / \mathrm{ml})$ and M-CSF $(10 \mathrm{ng} / \mathrm{ml})$ until multinucleated osteoclasts were formed. Then the cells were treated with or without FvMs in the presence of M-CSF and RANKL for 2 days. After the incubation, attached cells were completely removed from bone slices with mechanical agitation. Resorption pits on bone slices were visualized by staining the slices with Hematoxylin solution. To quantify the osteoclast bone resorption, pit areas were analyzed by image analysis (Paint.NET; http://www.getpaint. net/index.html).

Osteoclast survival and actin ring formation assays. Mature osteoclasts were treated with or without FvMs in the presence of M-CSF and RANKL for $24 \mathrm{~h}$. After washing twice with PBS, attached cells were stained for TRAP. Another set of cultures was assessed for actin ring formation assay. Briefly, cells were fixed in $4 \%$ paraformaldehyde and permeabilized with $0.1 \%$ Triton X-100. After washing with PBS, the cells were stained with TRITC-conjugated phalloidin (Sigma-Aldrich) or DAPI to visualize F-actin or nuclei, respectively. Fluorescence images were obtained by using a fluorescence microscope.

Animals and treatments. Thirty-two female C57BL/6 mice, 8 weeks of age, were purchased from the DaeHan Biolink (Seoul, Korea) and acclimated to laboratory conditions for 1 week before the experiment. Sham-operated (sham, $n=8$ ) mice were subjected to sham surgery exposure, but their ovaries were not removed. Ovariectomy (OVX, $\mathrm{n}=24$ ) was performed on the other groups by removing the bilateral ovaries and randomly assigning them to three groups ( $\mathrm{n}=8$ per group): the OVX, low dose of FvMs (30 mg/kg), and high dose of FvMs $(100 \mathrm{mg} / \mathrm{kg}$ ) groups. Mice were housed in an air-conditioned room with a 12-h light/dark cycle at a temperature of $22 \pm 2^{\circ} \mathrm{C}$ and $45-65 \%$ humidity, and given free access to food and tap water. Drug treatment started 10 days after surgery. Water was injected into negative control groups (sham and OVX). For the others, FvMs 30 (OVX+FvMs 30) or FvMs 100 mg/kg/day (OVX+FvMs 100) was administered orally for 6 weeks. At the end of treatment, all the mice were anesthetized using pentobarbitone, and exsanguination was performed by cardiac puncture followed by cervical dislocation. Serum was separated by centrifugation $(1,500 \mathrm{x} \mathrm{g})$ and then stored at $-80^{\circ} \mathrm{C}$ for future bone metabolic marker assay. The bilateral femurs were harvested, fixed with a $3.7 \%$ formaldehyde in PBS solution ( $\mathrm{pH}$ 7.4) for $16 \mathrm{~h}$, and then stored $\left(4^{\circ} \mathrm{C}\right)$ at $80 \%$ ethanol for microcomputed tomography.

Bone metabolic markers. Serum osteocalcin levels, the sensitive biomarker of bone formation, was measured by osteocalcin EIA kit (Biomedical Technologies, Stoughton, MA), and the effects of the treatments on bone resorption were evaluated using a RatLaps ${ }^{\mathrm{TM}}$ ELISA kit (Nordic Bioscience Diagnostics, Herlev, Denmark) to detect C-terminal telopeptide fragments of type I collagen C-terminus (CTX) generated by the osteoclasts (15).

Microcomputed tomography $(\mu C T)$. Microcomputerized tomography analysis $(\mu \mathrm{CT})$ of the femur was performed using the eXplore Locus SP scanner (GE Healthcare). The imaging parameters included exposure time $3 \mathrm{sec} /$ frame, X-ray energy settings of $80 \mathrm{kV}$ and current of $80 \mu \mathrm{A}$, a scan technique of $360^{\circ}$ rotation, and an isotopic resolution of $8 \times 8 \times 8 \mu \mathrm{m}$ voxel size. The image analyses were performed with the software Reconstruction Utility and Microview 2.2 (GE Healthcare). For the bone analysis, the region of 0.7-2.3 $\mathrm{mm}$ from the growth plate was selected as the region of interest (ROI) and image information was obtained based on the automatic domain value yielded by the computer. Several bone morphometric parameters, including bone mineral density (BMD), bone mineral content (BMC), bone volume fraction (BVF), tissue mineral density (TMD), trabecular separation (Tb.Sp.), and cortical bone mineral density (Cr.BMD) were obtained by analyzing the ROI. 
A

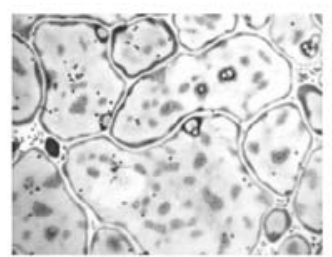

RANKL

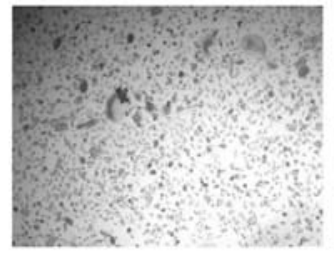

$\mathrm{R}+\mathrm{FvMs} 1$

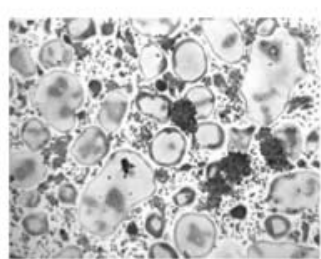

$\mathrm{R}+\mathrm{FvMs} 0.5$

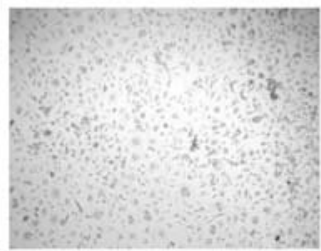

R+FvMs $2(\mu \mathrm{g} / \mathrm{ml})$

C

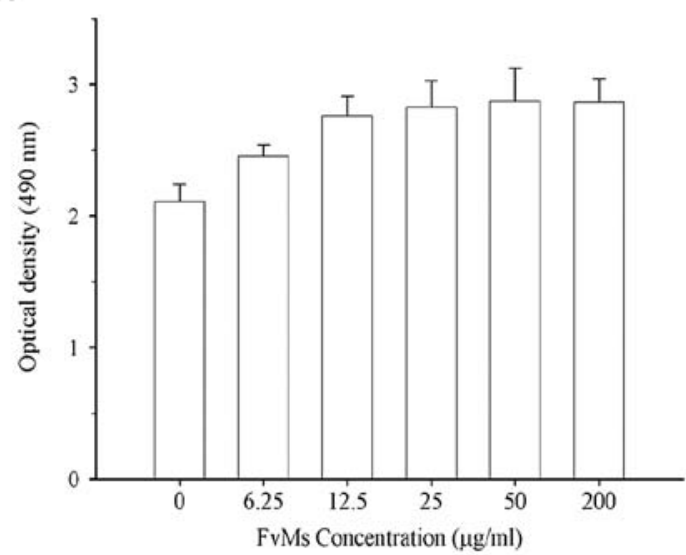

B

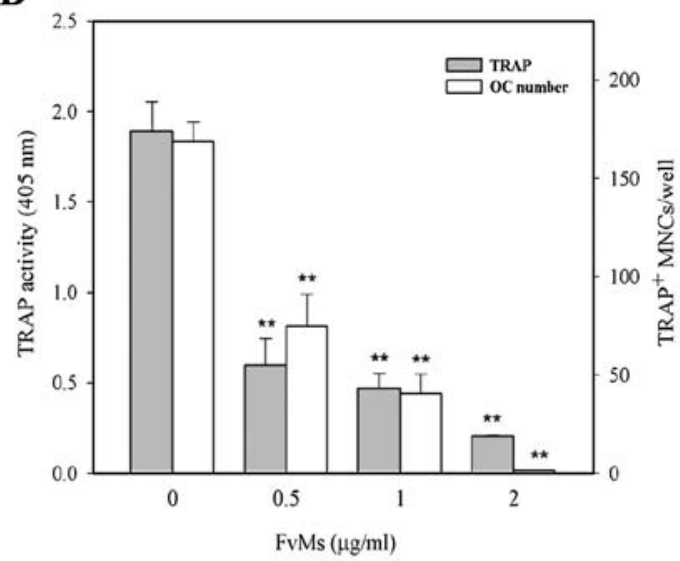

D

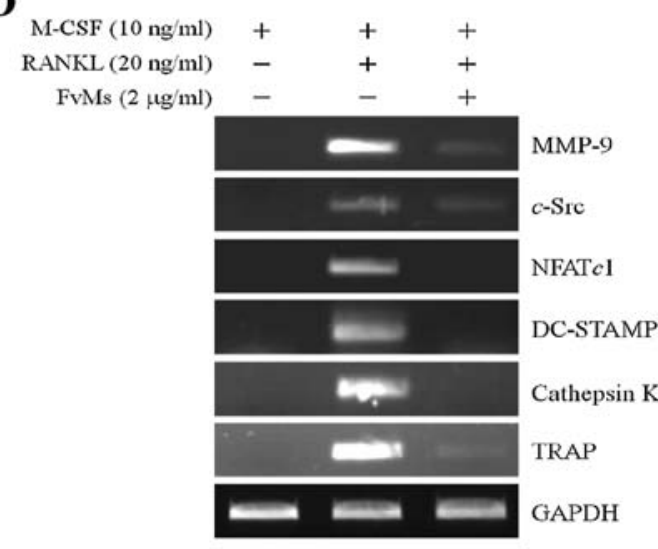

Figure 1. Effects of FvMs on RANKL-induced osteoclast differentiation in mouse bone-marrow-derived macrophages (BMMs). (A) Mouse BMMs were cultured with M-CSF, RANKL, and various concentration of FvMs. After OCs formed, the cells were fixed and stained for TRAP. (B) TRAP activity was measured at $405 \mathrm{~nm}$, and the TRAP+MNCs containing three or more nuclei were scored as osteoclasts. ${ }^{* *} \mathrm{P}<0.01$, significant differences from the control. (C) BMMs were cultured in the presence of M-CSF, RANKL, and FvMs for 2 days. Cell viability was measured by CellTiter 96 ${ }^{\circledR}$ AQueous One Solution Cell Proliferation Assay kit. Results are represented as mean \pm SD of three independent experiments. (D) BMMs were cultured with M-CSF in the absence or presence of RANKL with FvMs $(2 \mu \mathrm{g} / \mathrm{ml})$. RT-RCR was performed for the expression of osteoclastogenic markers at day 4.

Femoral mechanical testing. Mechanical femoral resistance was determined by a three-point bending test using the Universal Testing Machine (Instron 4202; Instron, Canton, MA). The detailed procedures were previously described (11).

Statistical analysis. The results were expressed as mean \pm S.D. Statistical significance was determined by the 2-tailed Student's t-test. The differences were considered to be significant at $\mathrm{P}<0.05$.

\section{Results}

Inhibition of RANKL-induced osteoclastogenesis and mRNA expression of osteoclast-specific genes by the aqueous extract of Foeniculum vulgare Miller seed ( $F v M s$ ). To evaluate whether the extracts have potential in alleviating osteoporosis-related diseases, we first collected 250 kinds of herbal plants, and prepared sample fractions by 5 solvents: aqueous, ethanolic, methanolic, ethyl acetate, or DMSO fractions, respectively. From amongst the extracts tested, the FvMs exhibited the most potent activity by TRAP assay (data not shown). To define the effective doses of FvMs on osteoclast formation of mouse
BMM cells, various concentrations of FvMs were applied to mouse BMM cells undergoing osteoclast differentiation. The FvMs inhibited osteoclast formation by 56,76 , and $99 \%$ at 0.5 , 1.0 , and $2.0 \mu \mathrm{g} / \mathrm{ml}$, respectively (Fig. 1). To ascertain that the inhibitory effects of FvMs on osteoclast formation were not due to its cytotoxicity, its effect on cell viability was evaluated. As shown in Fig. 1C, FvMs caused neither cytotoxicity nor reduced growth of osteoclast precursors within the concentrations tested in this study (up to $200 \mu \mathrm{g} / \mathrm{ml}$ ). Additionally, FvMs $(2.0 \mu \mathrm{g} / \mathrm{ml})$ treatment with RANKL significantly suppressed the expression of osteoclast-associated genes, including NFATc1, c-Src, DC-STAMP and TRAP which were dramatically induced by RANKL (Fig. 1D).

Differentiation of osteoclast precursors into osteoclasts consists of multiple steps, including cell adhesion, fusion, and differentiation. To evaluate which stages of osteoclastogenesis are targeted by FvMs, we added FvMs into BMM cultures treated with RANKL and M-CSF at different time points. Our result revealed that the FvMs targets the early stage of RANKL-induced osteoclast differentiation (data not shown). 
A

Control

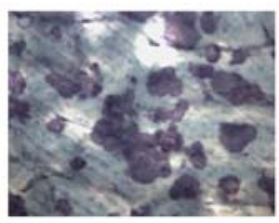

B

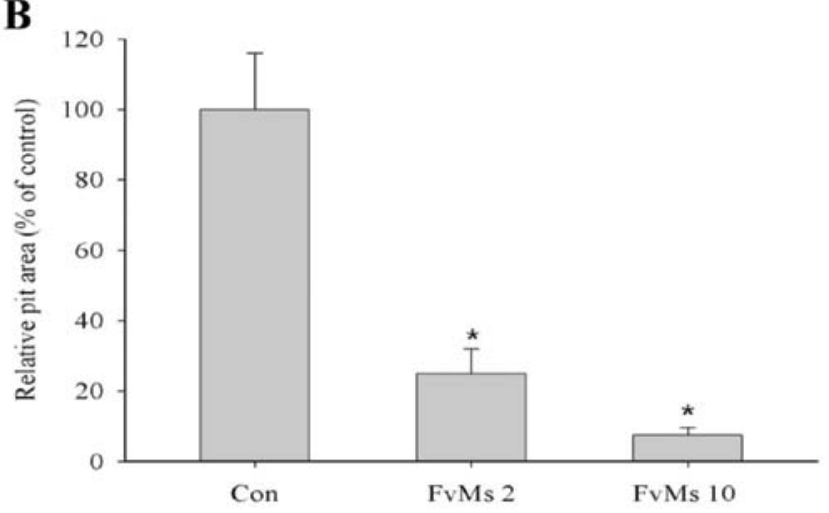

C
Control
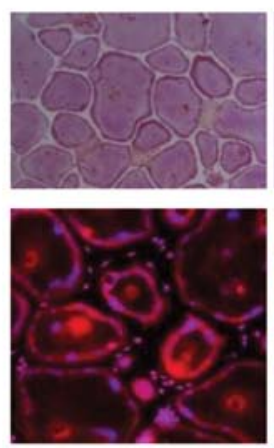

FvMs 2
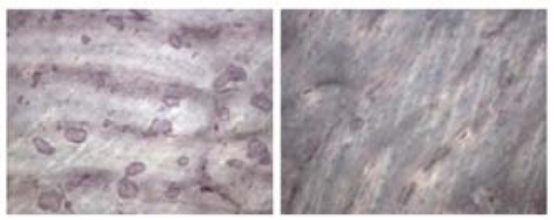

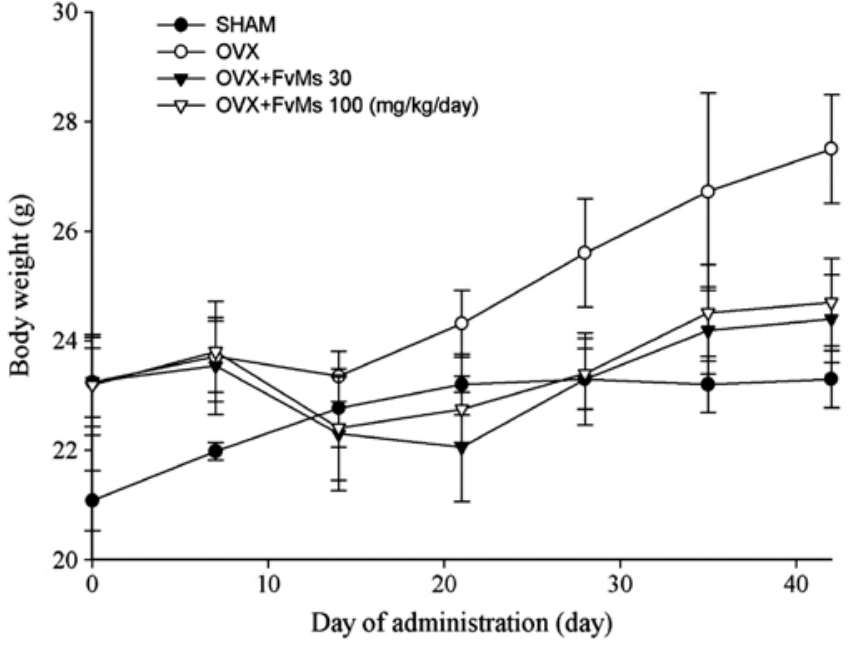

Figure 3. Changes in body weight during treatment. Data are reported as mean \pm SD. Symbols are: sham-operated $(\bullet)$, OVX group (O), OVXadministered $30 \mu \mathrm{g}$ FvMs $(\mathbf{\nabla})$, and $100 \mu \mathrm{g}$ group $(\nabla)$.

observed on the bone slices was not affected compared with the control group, however, their size was somewhat constricted and they were irregular in shape (Fig. 2C).

Effects of FvMs on bone loss in OVX-mice. At the end of the treatment, the mean final body weight was significantly increased in the OVX group $(26.72 \pm 1.80)$ compared with sham mice. Both the low-dose and high-dose FvMs groups had about $9 \%$ lower body weights $(\mathrm{p}<0.05)$ than the vehicletreated OVX group (Fig. 3). Fig. 4A shows the representative $\mu \mathrm{CT}$ images of distal femur metaphyses. The vehicle-treated OVX mice had lower values in BMD (-22.1\%), BMC (-26.8\%), TMD, and Cr.BMD, and a higher value in trabecular space (Tb.Sp) when compared to the vehicle-treated sham mice, differences that are statistically significance (Fig. 4B). Treatment with low doses of FvMs ( $30 \mathrm{mg} / \mathrm{kg} / \mathrm{day})$ caused a significant increase in BMC and BMD of trabecular bone in the femur, compared with OVX controls. TMD and Cr.BMD were also significantly increased compared with OVX mice. These results show that administration of FvMs improved BMD and BMC of the trabecular bone and decreased bone loss induced by OVX.

Mechanical strength analyses and serum bone markers. The OVX group showed on average 25\% lower maximal load than that of the sham group $(\mathrm{P}<0.05)$. Treatments with FvMs could prevent the deterioration of bending strength; the group treated with FvMs $(100 \mathrm{mg} / \mathrm{kg} / \mathrm{day})$ had significant higher values for maximal load (21\%) and strength (20\%) than the OVX control group (Fig. 5). On serum bone markers, as compared with the sham group, the OVX group showed 1.5 to 2-fold higher CTX and osteocalcin levels $(\mathrm{P}<0.05)$, respectively. Compared with vehicle-treated OVX animals, OVX mice treated with FvMs (30 or $100 \mathrm{mg} / \mathrm{kg} /$ day) had slightly (but not significantly) lower serum osteocalcin levels $(30 \%$ vs. OVX). In analysis of CTX, high doses of FvMs (100 mg/ $\mathrm{kg}$ /day) showed about a $10 \%$ decrease compared with the OVX group (data not shown). for optimal osteoclastic bone resorption. When osteoclasts with actin rings were treated with FvMs for $48 \mathrm{~h}$, the number of OCs
FvMs inhibits resorption pit formation in vitro. Differentiated surface. To examine whether FvMs inhibits osteoclast function, we performed the in vitro resorption pit assay. Mature osteoclasts were incubated on a dentine slice with M-CSF and RANKL in the presence or absence of FvMs ( 2 or $10 \mu \mathrm{g} / \mathrm{ml}$ ) for in a dose-dependent manner. The measurement of resorbed areas revealed more than $90 \%$ inhibition by $10 \mu \mathrm{g} / \mathrm{ml} \mathrm{FvMs}$ (Fig. 2A and B). Actin ring formation in osteoclasts is essential 
A

\begin{tabular}{ccc} 
& \multicolumn{3}{c}{ OVX } \\
\cline { 2 - 4 } SHAM & Con $\quad$ FvMs $30 \quad$ FvMs $100(\mathrm{mg} / \mathrm{kg} / \mathrm{day})$
\end{tabular}

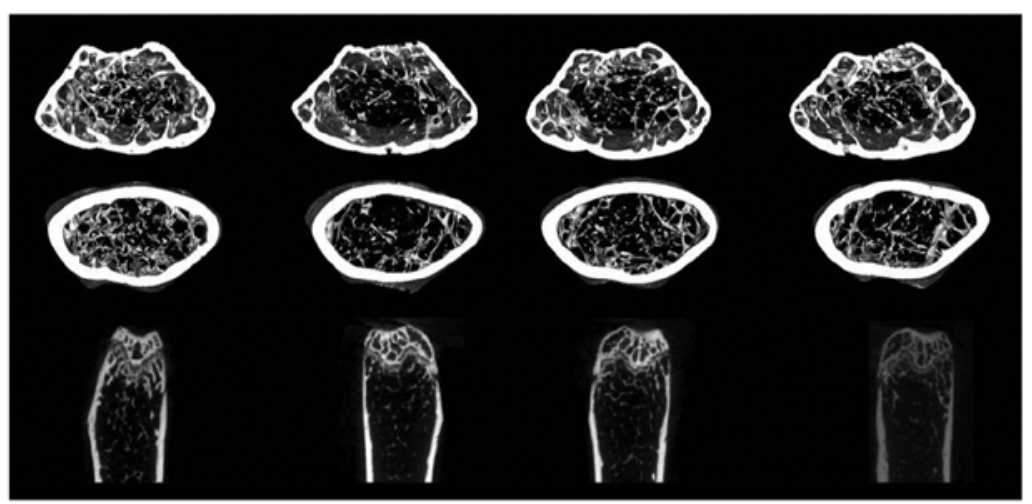

B
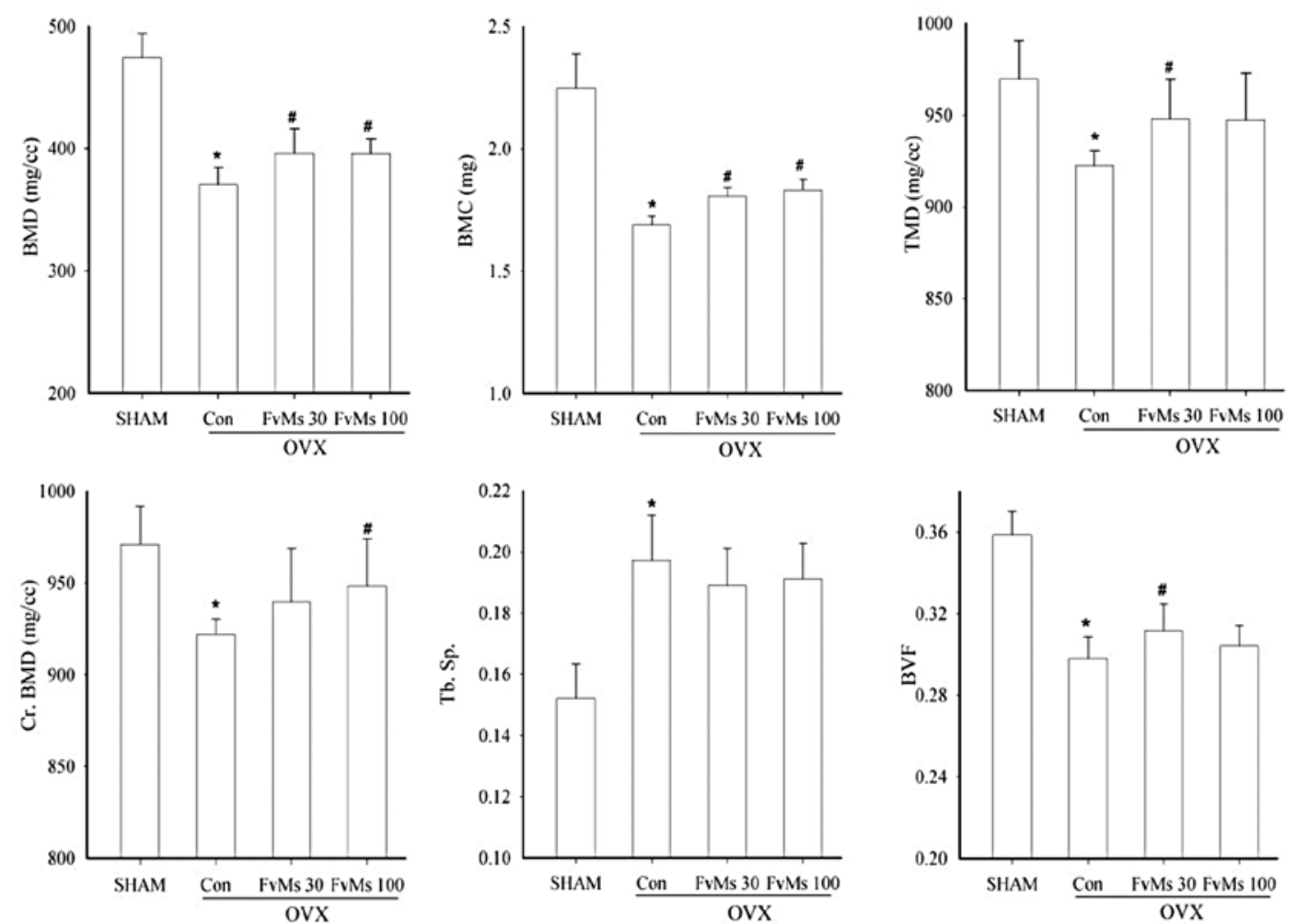

Figure 4. Representative $\mu \mathrm{CT}$ images of the distal femurs in each group. Histomorphometric analysis of distal femurs in mice treated with FvMs at doses of 30 or $100 \mathrm{mg} / \mathrm{kg} /$ day for 6 weeks after OVX. Bone mineral density (BMD), bone mineral content (BMC), bone volume fraction (BVF), tissue mineral density (TMD), trabecular separation (Tb.Sp.), and cortical bone mineral density (Cr.BMD). Results are shown as mean \pm SD. ${ }^{*} \mathrm{P}<0.05$ vs. sham control; ${ }^{\#} \mathrm{P}<0.05$ vs. OVX.

\section{Discussion}

Many plant-derived products have been used as folk medicines for bone-destructive diseases such as osteoporosis, periodontal diseases, and rheumatoid arthritis. Osteoclasts are multinucleated cells that have a unique role in bone degradation. In a search for natural compounds that suppress osteoclast formation and activity, an aqueous extract of Foeniculum vulgare Miller seed (FvMs) was identified as an effective agent. Foeniculum vulgare Miller (Fennel) has traditionally been used as a treatment for a variety of ailments, especially those of the digestive system (8). Recently, fennel has shown anti- cancer $(16,17)$, anti-inflammatory (7), and antioxidant activity $(8,9)$, and its oil fraction may have antidiabetic activity $(18)$.

Our study shows that FvMs dramatically inhibited TRAP activity and multinucleated cell formation in BMM cells (Fig. 1A and B) and in RAW264.7 cells (data not shown) while eliciting no cytotoxic responses (Fig. 1C). During the differentiation process, osteoclast specific genes, such as NFATc1, DC-STAMP, MMP-9, and TRAP were specifically induced by RANKL. In the BMM culture system, expression of those genes was abolished or greatly attenuated in the presence of FvMs (Fig1. D). NFATc1 is a key transcription factor for the expression of TRAP and other osteoclastogenesis-associated 
A

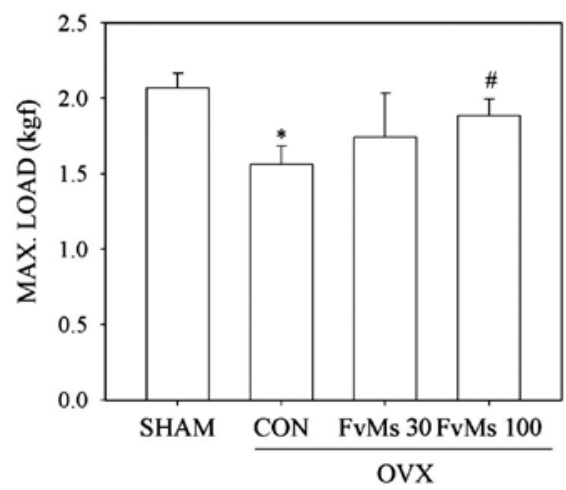

B

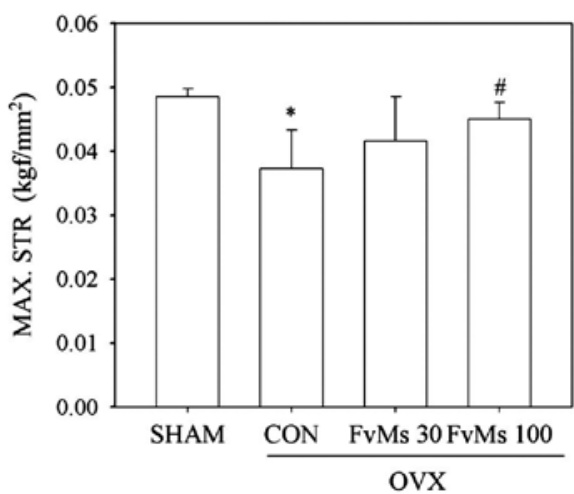

Figure 5. Evaluations of midshaft femur biomechanical properties by using a three-point bending test. Loading force to maximal load (A), and maximal strength (B). Ovariectomy significantly decreased maximal load and strength as compared with the sham group; however, the $100 \mathrm{mg} / \mathrm{kg} / \mathrm{day}$ of FvMs group showed effects to counteract the deteriorations of these parameters. ${ }^{*} \mathrm{P}<0.05$ vs. sham control; ${ }^{\#} \mathrm{P}<0.05$ vs. OVX.

genes (19). Furthermore, FvMs strongly inhibited RANKLinduced osteoclast formation when added during the early stages of culture. In the middle and terminal stage of the culture, the inhibitory effect of FvMs on osteoclast formation was attenuated, suggesting that FvMs acts more strongly on osteoclast precursors (data not shown).

Bone resorbing osteoclasts form the actin ring structure, which is considered essential for bone resorption by activated osteoclasts (20). Therefore, finding drugs that disturb the integrity of the actin ring could be a useful approach to therapy for reducing bone resorption. In this experiment, FvMs inhibited bone resorption in a dose-dependent manner (Fig. 2). Although their size and shape were somewhat different between groups, the number of OCs observed on the bone slices was not affected compared with the control group. The regulation of osteoclast survival is an important mechanism in the regulation of mature osteoclast bone-resorbing activity. We assumed that the inhibitory effect of FvMs on bone resorption is partially due to apoptosis of mature osteoclasts; however, in our experiments mature osteoclast survival was not affected by treatment with FvMs. Therefore, these results suggest that mature OC apoptosis or disrupted actin ring formation is not the main inhibitory pathway of FvMs on osteoclastic bone resorption.

Estrogen deficiency resulting from OVX leads to an increased rate of bone turnover and an imbalance between bone resorption and formation (21). Since high turnover may lead to bone loss, downregulation of bone turnover may be beneficial to bone metabolism. As expected, OVX greatly reduced BMD in the distal femur resulting from increased bone turnover, as indicated by the higher plasma OC and CTX concentrations in the OVX group compared to the sham group (Fig. 4). These results are in agreement with those from Wronski et al (22), which demonstrated that bone remodeling is accelerated after the cessation of ovarian function. Our results showed that FvMs slightly decreased bone turnover markers that were accelerated by OVX. The mechanical properties of the bone are influenced by bone mass as well as bone size, bone quality, and cancellous bone architecture (23). Our experimental data demonstrate that FvMs attenuates estrogen-dependent bone loss and biomechanical deterioration in OVX mice in a dosedependent manner (Figs. 4 and 5).
Estrogen deficiency induces an unregulated chronic inflammatory process by increasing the local production of various osteoclastogenic cytokines within the bone microenvironment (24). One of the cytokines responsible for the enhanced osteoclastogenesis in states of estrogen deficiency is TNF- $\alpha$. It induces osteoclast formation by upregulating stromal cell production of RANKL and M-CSF (25). In addition, administration of $\mathrm{N}$-acetyl-cysteine or ascorbate antioxidants decreased osteoclast numbers on the bone surface, thereby abolishing OVX-induced bone loss (26). Therefore, application of antioxidant or anti-inflammatory compounds, or compounds that are both antioxidant and anti-inflammatory, may be a beneficial alternative therapy for bone diseases.

In summary, the present study demonstrated that FvMs suppresses the osteoclast differentiation and bone resorption of mature osteoclasts. In addition, it prevents the decrease of bone mass induced by OVX, especially in trabecular bone, and enhances bone strength. Therefore, Foeniculum vulgare Miller may be a beneficial natural resource for prevention or treatment of bone loss induced by estrogen deficiency.

\section{Acknowledgements}

This study was supported by a grant of Korea Health Technology R\&D Project from the Ministry for Health and Welfare, Republic of Korea (A010252).

\section{References}

1. Parfitt AM: Bone remodeling and bone loss: understanding the pathophysiology of osteoporosis. Clin Obstet Gynecol 30: 789-811, 1987.

2. Boyle WJ, Simonet WS and Lacey DL: Osteoclast differentiation and activation. Nature 423: 337-342, 2003.

3. Weitzmann MN and Pacifici R: Estrogen deficiency and bone loss: an inflammatory tale. J Clin Invest 116: 1186-1194, 2006.

4. Rodan GA and Martin TJ: Therapeutic approaches to bone diseases. Science 289: 1508-1514, 2000.

5. Reid IR: Pharmacotherapy of osteoporosis in postmenopausal women: focus on safety. Expert Opin Drug Saf 1: 93-107, 2002.

6. Yeh IT: Postmenopausal hormone replacement therapy: endometrial and breast effects. Adv Anat Pathol 14: 17-24, 2007.

7. Choi EM and Hwang JK: Antiinflammatory, analgesic and antioxidant activities of the fruit of Foeniculum vulgare. Fitoterapia 75: 557-565, 2004. 
8. Barros L, Heleno SA, Carvalho AM and Ferreira IC: Systematic evaluation of the antioxidant potential of different parts of Foeniculum vulgare Mill. from Portugal. Food Chem Toxicol 47: 2458-2464, 2009.

9. Nickavar B and Abolhasani FA: Screening of antioxidant properties of seven Umbelliferae fruits from Iran. Pak J Pharm Sci 22: 30-35, 2009.

10. Ha BG, Hong JM, Park JY, Ha MH, Kim TH, Cho JY, Ryoo HM, Choi JY, Shin HI, Chun SY, Kim SY and Park EK: Proteomic profile of osteoclast membrane proteins: identification of $\mathrm{Na}^{+} / \mathrm{H}^{+}$ exchanger domain containing 2 and its role in osteoclast fusion. Proteomics 8: 2625-2639, 2008.

11. Kim TH, Jung JW, Ha BG, Hong JM, Park EK, Kim HJ and Kim SY: The effects of luteolin on osteoclast differentiation, function in vitro and ovariectomy-induced bone loss. J Nutr Biochem 22: 8-15, 2011.

12. Faccio R, Takeshita S, Zallone A, Ross FP and Teitelbaum SL: c-Fms and the alphavbeta3 integrin collaborate during osteoclast differentiation. J Clin Invest 111: 749-758, 2003.

13. Battaglino R, Kim D, Fu J, Vaage B, Fu XY and Stashenko P. $\mathrm{c}-\mathrm{Myc}$ is required for osteoclast differentiation. $\mathrm{J}$ Bone Miner Res 17: 763-773, 2002.

14. Yagi M, Ninomiya K, Fujita N, Suzuki T, Iwasaki R, Morita K, Hosogane N, Matsuo K, Toyama Y, Suda T and Miyamoto T: Induction of DC-STAMP by alternative activation and downstream signaling mechanisms. J Bone Miner Res 22: 992-1001, 2007.

15. Meli R, Pacilio M, Raso GM, Esposito E, Coppola A, Nasti A Di Carlo C, Nappi C and Di Carlo R: Estrogen and raloxifene modulate leptin and its receptor in hypothalamus and adipose tissue from ovariectomized rats. Endocrinology 145: 3115-3121, 2004.

16. Singh B and Kale RK: Chemomodulatory action of Foeniculum vulgare (Fennel) on skin and forestomach papillomagenesis, enzymes associated with xenobiotic metabolism and antioxidant status in murine model system. Food Chem Toxicol 46: 3842-3850, 2008.

17. Celik I and Isik I: Determination of chemopreventive role of Foeniculum vulgare and Salvia officinalis infusion on trichloroacetic acid-induced increased serum marker enzymes lipid peroxidation and antioxidative defense systems in rats. Nat Prod Res 22: 66-75, 2008.
18. Shahat AA, Ibrahim AY, Hendawy SF, Omer EA, Hammouda FM, Abdel-Rahman FH and Saleh MA: Chemical composition, antimicrobial and antioxidant activities of essential oils from organically cultivated fennel cultivars. Molecules 16: 1366-1377, 2011.

19. Takayanagi H, Kim S, Koga T, Nishina H, Isshiki M, Yoshida H, Saiura A, Isobe M, Yokochi T, Inoue J, Wagner EF, Mak TW, Kodama $\mathrm{T}$ and Taniguchi T: Induction and activation of the transcription factor NFATc1 (NFAT2) integrate RANKL signaling in terminal differentiation of osteoclasts. Dev Cell 3: 889-901, 2002.

20. Suda T, Nakamura I, Jimi E and Takahashi N: Regulation of osteoclast function. J Bone Miner Res 12: 869-879, 1997.

21. Raisz LG: Pathogenesis of osteoporosis: concepts, conflicts, and prospects. J Clin Invest 115: 3318-3325, 2005

22. Wronski TJ, Lowry PL, Walsh CC and Ignaszewski LA: Skeletal alterations in ovariectomized rats. Calcif Tissue Int 37: 324-328, 1985.

23. Kinney JH, Haupt DL, Balooch M, Ladd AJ, Ryaby JT and Lane NE: Three-dimensional morphometry of the L6 vertebra in the ovariectomized rat model of osteoporosis: biomechanical implications. J Bone Miner Res 15: 1981-1991, 2000.

24. Notoya M, Arai R, Katafuchi T, Minamino N and Hagiwara H: A novel member of the calcitonin gene-related peptide family, calcitonin receptor-stimulating peptide, inhibits the formation and activity of osteoclasts. Eur J Pharmacol 560: 234-239, 2007.

25. Hotokezaka H, Sakai E, Ohara N, Hotokezaka Y, Gonzales C, Matsuo K, Fujimura Y, Yoshida N and Nakayama K: Molecular analysis of RANKL-independent cell fusion of osteoclast-like cells induced by TNF-alpha, lipopolysaccharide, or peptidoglycan. J Cell Biochem 101: 122-134, 2007.

26. Lean JM, Davies JT, Fuller K, Jagger CJ, Kirstein B, Partington GA, Urry ZL and Chambers TJ: A crucial role for thiol antioxidants in estrogen-deficiency bone loss. J Clin Invest 112: 915-923, 2003. 